\title{
Consumo de agrotóxicos e distribuição temporal da proporção de nascimentos masculinos no Estado do Paraná, Brasil
}

\author{
Gerusa Gibson ${ }^{1}$ e Sergio Koifman ${ }^{1}$
}

Como citar Gibson G, Koifman S. Consumo de agrotóxicos e distribuição temporal da proporção de nascimentos masculinos no Estado do Paraná, Brasil. Rev Panam Salud Publica. 2008;24(4):240-7.

RESUMO Objetivo. Investigar a tendência temporal da proporção de nascimentos masculinos para o Estado do Paraná no período entre 1994 e 2004 e a correlação dessa tendência com o volume de vendas de agrotóxicos no Estado em 1985.

Método. No presente estudo ecológico, foram empregados como fontes de dados o Instituto Brasileiro de Geografia e Estatística (volume de vendas de agrotóxicos) e o banco DATASUS (nascidos vivos segundo o sexo). Foram analisados 308 municípios, divididos em quartis conforme o consumo de agrotóxicos, resultando em quatro estratos contendo 77 municípios cada um, sendo o quarto quartil o de maior consumo. A proporção de nascimentos masculinos foi obtida ano a ano para cada município e, ao final do período (1994 a 2004), um valor mediano foi calculado para cada uma das localidades. Foi realizada uma análise bivariada e calculados os coeficientes de Pearson para cada quartil de municípios. Uma análise exploratória e comparativa dos quartis foi realizada, na qual se calculou o consumo médio per capita para os mesmos. Posteriormente, foram selecionados e analisados individualmente 10 municípios com intensa atividade agropecuária quanto ao perfil agrícola e à tendência da proporção de nascimentos masculinos ao longo do período de estudo.

Resultados. Foi observada uma discreta tendência de declínio na proporção de nascidos vivos do sexo masculino para o Estado como um todo. A magnitude desse declínio foi visivelmente maior quando se analisou individualmente o grupo de 10 municípios, cujos valores observados ao final da série mostraram-se muito abaixo daqueles normalmente descritos na literatura (proporção de nascimentos masculinos < 50\%). Em relação aos quartis, todos apresentaram correlação negativa, ainda que sem significância estatística.

Conclusões. A tendência de declínio estatisticamente significativa na proporção de nascimentos masculinos em alguns municípios paranaenses sugere que o fenômeno possa ser decorrente da alta exposição ambiental aos agrotóxicos nessas localidades. Sendo assim, recomendam-se estudos futuros que visem a esclarecer e a dimensionar a força do impacto da contaminação ambiental por agrotóxicos na saúde reprodutiva, representada aqui pela razão de sexos ao nascimento.

Palavras-chave Agrotóxicos, poluição ambiental, saúde reprodutiva, Brasil.

1 Fundação Instituto Oswaldo Cruz, Escola Nacional de Saúde Pública, Departamento de Epidemiologia e Métodos Quantitativos em Saúde. Enviar correspondência para Gerusa Gibson no seguinte endereço: Rua Leopoldo Bulhões 1480/607, CEP 21041-210, Rio de Janeiro, RJ, Brasil. E-mail: gibson ge@ensp.fiocruz.br
Alguns agrotóxicos fazem parte de um conjunto de poluentes ambientais definidos como desreguladores hormonais. Tais compostos são capazes de alterar a fisiologia do sistema endó- crino a partir de múltiplos mecanismos, em especial pela capacidade de mimetização de hormônios verdadeiros, devido a semelhanças entre as suas estruturas moleculares (1). Se- 
gundo a literatura científica, a exposição crônica a esse conjunto de poluentes pode resultar, a longo prazo, em uma série de agravos de saúde relacionados a disfunções no sistema endócrino, a exemplo das neoplasias hormônio-dependentes e dos distúrbios da saúde reprodutiva, como a redução da fertilidade masculina, os abortos espontâneos e as malformações congênitas $(2,3)$.

A exposição a desreguladores endócrinos parece estar associada também à razão de sexos ao nascimento (4). Essa medida padrão, utilizada para avaliar a relação quantitativa entre os sexos, vem se mostrando sensível a condições ambientais adversas, apresentando tendência de declínio frente a tais condições. Sugere-se que a redução do número de nascimentos masculinos seja resultado da influência das concentrações hormonais dos pais no momento da concepção sobre o sexo do embrião a ser desenvolvido (1).

Os valores da razão de sexos ao nascimento descritos na literatura giram em torno de 1,06, ou seja, 106 nascimentos masculinos para cada 100 nascimentos femininos. No entanto, tal medida pode ser também referida pela proporção de nascimentos masculinos em relação ao total de nascidos vivos, padrão este adotado no presente trabalho. Nesse caso, a proporção usual de nascimentos do sexo masculino fica em torno de 51,5\% (5).

Em países com nível elevado de compostos com atividade endócrina no ambiente, tem sido relatada uma redução significativa de nascimentos de homens (6-9). Esse mesmo efeito já foi constatado em diversos estudos que avaliaram episódios de exposição aguda acidental ou ocupacional a essas substâncias (10-12). Tal observação tem levado muitos autores a considerar a razão de sexos ao nascimento como um indicador sentinela da exposição ambiental a compostos com atividade endócrina (4).

Em geral, os fatores que determinam a razão de sexos ao nascimento são classificados em duas categorias: aqueles que determinam a razão de sexos primária, ou seja, que influenciam o sexo na hora da concepção; e fa- tores secundários que, uma vez definido o sexo da criança, influenciam a sobrevivência do embrião no útero, estando, portanto, relacionados à incidência de natimortos (1).

Dentre os fatores que determinam a razão de sexos primária, destacam-se as concentrações hormonais dos pais no momento da concepção. Nesse momento, altos níveis de estrogênio e testosterona poderiam aumentar a probabilidade de nascimento de uma criança do sexo masculino, enquanto altas concentrações de gonadotrofina e progesterona parecem elevar a probabilidade de nascimentos femininos (1). Outros exemplos relatados na literatura mencionam ainda a idade do ovócito ao nascimento, a idade dos pais, a etnia e as diferenças na motilidade e no tempo de vida de espermatozóides $X$ e $Y(9,13)$.

Atualmente, sabe-se que agrotóxicos como o dibromopropano, dibromito de etileno e clordecone provocam efeitos tóxicos relevantes sobre a reprodução, afetando, sobretudo, a espermatogênese humana. No caso do dicloro-difenil-tricloroetano (DDT), foi constatado que o seu principal metabólito, o dicloro-difenil-dicloroeteno (DDE), é capaz de interferir na ação dos hormônios masculinos, promovendo um efeito feminizador (14).

Lemaire et al. (15) testaram a interação in vitro de pesticidas organoclorados com receptores andrógenos (hAR) em uma linhagem de células de próstata (PALM) e concluíram que a atividade endócrina dessas substâncias interfere na via de sinalização hormonal masculina. Esse fato reforça a hipótese de que o recente aumento na incidência de distúrbios sexuais masculinos pode ser conseqüência da exposição a longo prazo a pesticidas organoclorados presentes no ambiente. Outro estudo avaliou a situação dos níveis de testosterona em ratos expostos à atrazina, um dos agrotóxicos mais utilizados. Os resultados demonstraram uma ação disruptora sobre o sistema endócrino em ratos machos através da inibição direta da síntese de testosterona pelas células de Leydig nos testículos (16). Em outro trabalho, a exposição a desreguladores endócrinos ambientais foi associada a alterações nas características do sêmen e ao aumento das concentrações plasmáticas de hormônio folículo estimulante (FSH) e luteinizante (LH) nos grupos de homens estudados (17).

O crescente número de notificações de distúrbios reprodutivos em seres humanos e animais, associado à exposição a desreguladores endócrinos ambientais, reforça a hipótese de que esses contaminantes seriam responsáveis pelo aumento da incidência de agravos de saúde reprodutiva $(3,10$, $17,18)$. Sob esse aspecto, questiona-se o impacto da contaminação por agrotóxicos, cujos reflexos parecem resultar na redução de nascimentos de homens nas populações expostas (10). No contexto mundial, sabe-se que a proporção de nascimentos masculinos vem declinando em alguns países europeus, como Inglaterra e País de Gales (7), Dinamarca (8), Canadá (9) e Finlândia (14). Acredita-se que o aumento da exposição ambiental a desreguladores endócrinos nos últimos anos tenha contribuído para esse fato (10).

Considerando tais evidências, a hipótese de que haja um declínio na proporção de nascidos vivos do sexo masculino no Brasil torna-se claramente plausível, sobretudo se considerarmos a trajetória da agricultura brasileira, cujo modelo de desenvolvimento se baseia na crescente demanda por agrotóxicos e fertilizantes. Nesse contexto, o Estado do Paraná se destaca por ser um grande representante desse modelo de produção (19). O uso abusivo de agrotóxicos e fertilizantes, associado ao manejo inadequado dos solos, resulta em contaminação de águas e na erosão de solos com conseqüente desequilíbrio de cadeias tróficas e perda de biodiversidade (14). Devido à alta persistência e à mobilidade desses poluentes químicos através das diferentes matrizes ambientais, causando desequilíbrios ao ecossistema e agravos à saúde das populações expostas, o uso massivo de agrotóxicos se tornou um sério problema de saúde pública $(14,20)$.

Nesse sentido, o objetivo do presente trabalho foi analisar a distribuição temporal da proporção de nasci- 
mentos masculinos no Estado do Paraná no período de 1994 a 2004, assim como avaliar a natureza da correlação existente entre a tendência observada e o volume de vendas de agrotóxicos segundo municípios em 1985, utilizado aqui como indicador de exposição.

\section{MATERIAIS E MÉTODOS}

O presente estudo ecológico buscou explorar a associação entre o consumo de agrotóxicos no Estado do Paraná e possíveis alterações na distribuição temporal da proporção de nascimentos masculinos.

Foram utilizados os dados do Instituto Brasileiro de Geografia e Estatística (IBGE) referentes ao volume de vendas de agrotóxicos em moeda local no ano de 1985 e ao perfil agrícola dos municípios. As informações relativas ao nascimento segundo sexo para o período de 1994 a 2004 foram obtidas do banco de dados do Sistema Único de Saúde (DATASUS). Como é necessário um longo período de exposição crônica aos agrotóxicos para que os impactos na proporção de sexos ao nascimento sejam perceptíveis, foram estudados os nascimentos ocorridos a partir de 8 anos após o ano considerado para o consumo de agrotóxicos (neste caso, o ano de consumo foi 1985, e as séries utilizadas foram de 1994 a 2004).

Dos 399 municípios paranaenses oficialmente cadastrados no IBGE, foram excluídos aqueles que sofreram modificações em seus territórios no período de estudo, assim como aqueles cujas informações sobre venda de agrotóxicos em moeda local no ano de 1985 não estavam disponíveis, restando, assim, 308 municípios elegíveis.

\section{Correlação bivariada}

Para a análise de correlação, foi utilizado o Statistical Package for the Social Sciences (SPSS) versão 9.0. As variáveis utilizadas nessa etapa foram o consumo de agrotóxicos (volume de venda em cruzeiros, moeda nacional da época) nos 308 municípios elegíveis do Paraná em 1985 e os valores medianos das proporções de nascimentos masculinos para o período de 1994 a 2004 . O banco de dados referente ao consumo de agrotóxicos foi dividido em quatro estratos contendo 77 municípios cada um. Os pontos de corte para o consumo de agrotóxicos foram: Cr\$44,50 per capita para os municípios pertencentes ao primeiro quartil; até 125,0 para o segundo quartil; até 239,0 para o terceiro quartil; e até 1195,7 para o quarto quartil, que engloba os municípios com maior consumo.

A análise de correlação foi realizada separadamente dentro de cada quartil de consumo. Os valores da variável proporção de nascimentos masculinos foram obtidos ano a ano para cada município e, ao final do período, um valor mediano foi calculado para cada uma das localidades. Optou-se por utilizar a mediana ao invés da média dos valores anuais da proporção de nascidos vivos do sexo masculino para evitar a interferência de valores eventuais incomuns (outliers) no valor final da variável. Nesta etapa, optou-se também por utilizar o consumo de agrotóxicos per capita, de maneira que a exposição estivesse representada de forma mais fidedigna, proporcional ao número de habitantes de cada município.

Foram calculados os coeficientes de Pearson para a correlação entre a proporção de nascimentos masculinos e o consumo de agrotóxicos em cada quartil de municípios. Posteriormente, construiu-se um diagrama de dispersão para o quarto quartil, estrato de maior consumo.

\section{Análise exploratória dos dados}

Foi realizada uma análise descritiva e comparativa dos quartis. Nessa análise, foi calculado o consumo médio per capita de agrotóxicos por quartil (em cruzeiros, moeda nacional da época), bem como a mediana dos valores das proporções de nascimentos masculinos para cada um dos quartis referente ao período entre 1994 e 2004. Foram selecionados, aleatoriamente, 10 municípios de pequeno porte e intensa atividade agrícola para análise individual da tendência temporal da proporção de nascimentos masculinos. Considerando as grandes diferenças regionais dentro do Estado do Paraná, a análise individual de municípios essencialmente agrícolas reproduz de forma mais fidedigna os possíveis efeitos da exposição a agrotóxicos.

\section{Análise da tendência}

Optou-se pela utilização de modelos de regressão polinomial em virtude do poder estatístico bem como pela facilidade de formulação e interpretação que os mesmos oferecem. O modelo de regressão polinomial tem por objetivo encontrar a equação que melhor descreve a relação existente entre a variável independente $X$ (ano de consumo de agrotóxicos) e a variável dependente Y (proporção de nascidos vivos do sexo masculino). Nesta etapa, tal modelo foi utilizado para analisar a tendência da proporção de nascimentos masculinos para o Estado do Paraná e os 10 municípios selecionados.

Foi estabelecido um nível de significância de $\alpha<0,05$. O programa utilizado para a elaboração dos modelos de regressão polinomial e dos gráficos de dispersão foi o SPSS 9.0.

\section{Descrição espacial dos dados}

A análise espacial foi realizada no programa TerraVieww versão 3.1.4 (http:/ / www.terraview.org/), utilizando-se o estimador de intensidade Kernel, uma ferramenta que permite estimar a quantidade de eventos por unidade de área em cada célula de uma grade regular que recobre a região estudada. O estimador de Kernel é uma técnica não paramétrica que promove alisamento ou suavização estatística e que possibilita filtrar a variabilidade de um conjunto de dados, preservando as características essenciais dos locais. A escolha de um parâmetro definido como largura de banda é que determina o grau de alisamento. Esse parâmetro indica a área a ser considerada no cálculo, devendo ainda refletir a escala geográfica da hipótese de interesse. Na presente análise, foi utilizada 
a função de suavização quártica com raio adaptativo para o Kernel referente ao consumo de agrotóxicos.

\section{RESULTADOS}

Os coeficientes de Pearson para a correlação entre o consumo de agrotóxicos e a tendência da proporção de nascidos vivos do sexo masculino foram de $-0,080,-0,149,-0,098$ e $-0,125$, respectivamente, para o primeiro, segundo, terceiro e quarto quartis; não foi detectada significância estatística (tabela 1). Os diagramas de dispersão correspondentes aos quartis corroboram os valores dos coeficientes de Pearson encontrados, evidenciando uma correlação negativa próxima de zero para os municípios dos três primeiros quartis (gráficos não apresentados) e quarto quartil (figura 1A).

Foi observada uma discreta tendência de declínio da proporção de nasci-

TABELA 1. Correlação bivariada entre o consumo per capita de agrotóxicos em 1985 e as medianas das proporções de nascimentos masculinos por quartila no período de 1994 a 2004, Estado do Paraná, Brasil

\begin{tabular}{lccccc}
\hline & $\begin{array}{c}\text { Consumo de } \\
\text { agrotóxicos } \\
(\text { em moeda } \\
\text { da época) }\end{array}$ & $\begin{array}{c}\text { Consumo per } \\
\text { capita de } \\
\text { agrotóxicos } \\
(\text { em moeda } \\
\text { da época) }\end{array}$ & $\begin{array}{c}\text { Proporção de } \\
\text { nascimentos } \\
\text { masculinos } \\
(\%)^{c}\end{array}$ & $\begin{array}{c}\text { Coeficiente } \\
\text { de Pearson }\end{array}$ & $P$ \\
\hline Quartila & 665200,6 & 14,0 & 50,9 & $-0,080$ & 0,487 \\
Segundo & 2089287,0 & 82,5 & 50,5 & $-0,149$ & 0,196 \\
Terceiro & 2754912,6 & 177,6 & 51,9 & $-0,098$ & 0,395 \\
Quarto & 5874764,2 & 429,5 & 50,5 & $-0,125$ & 0,277 \\
\hline
\end{tabular}

a 0 consumo de agrotóxicos nos 308 municípios foi dividido em quartis contendo 77 municípios cada um. 0 quarto quartil é 0 de maior consumo.

b Para 0 ano de 1985 segundo o IBGE.

c Para o período de 1994 a 2004 segundo o DATASUS.

mentos masculinos para o Estado do Paraná no período, no entanto, sem significância estatística (figura 2). Este efeito é mais evidente quando são analisados separadamente os municípios de Santo Antônio do Caiuá, Nova
Aliança do Ivaí, Jardim Olinda, Guairaçá, Icaraíma, Doutor Camargo, Rio Bom, Cafeara, Palmeira e Barra do Jacaré, caracterizados pelo pequeno porte (média de 10000 habitantes) e intensa atividade agrícola.

FIGURA 1. Diagrama de dispersão entre o consumo de agrotóxicos em 1985 e a tendência da proporção de nascimentos masculinos de 1994 a 2004, Estado do Paraná, Brasil
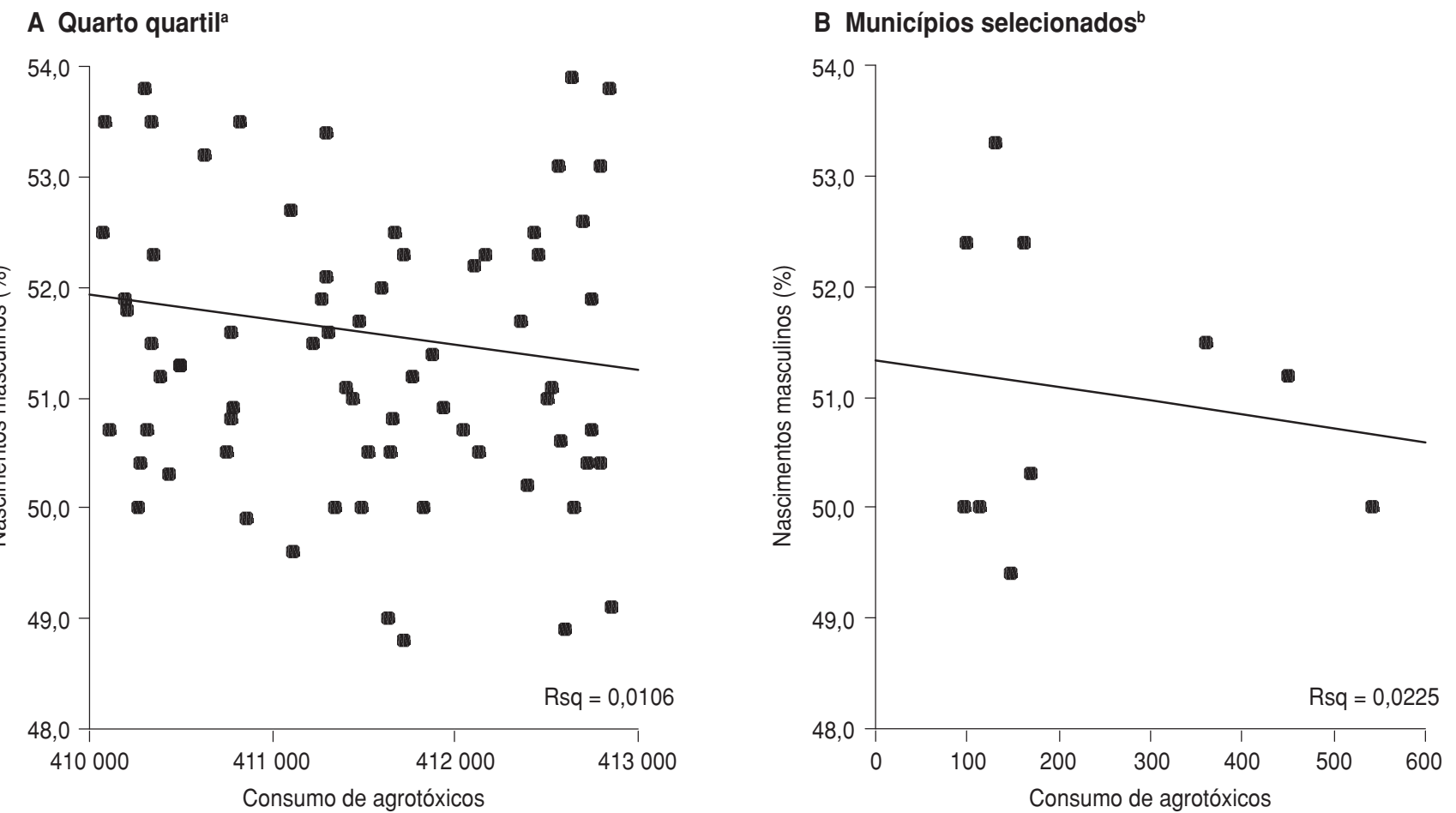

a Os municípios foram divididos em quartis de consumo de agrotóxicos contendo 77 municípios cada um. O quarto quartil engloba os municípios com o maior consumo.

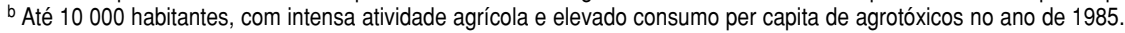


FIGURA 2. Tendência temporal da proporção de nascimentos masculinos no Estado do Paraná, Brasil, 1994 a 2004

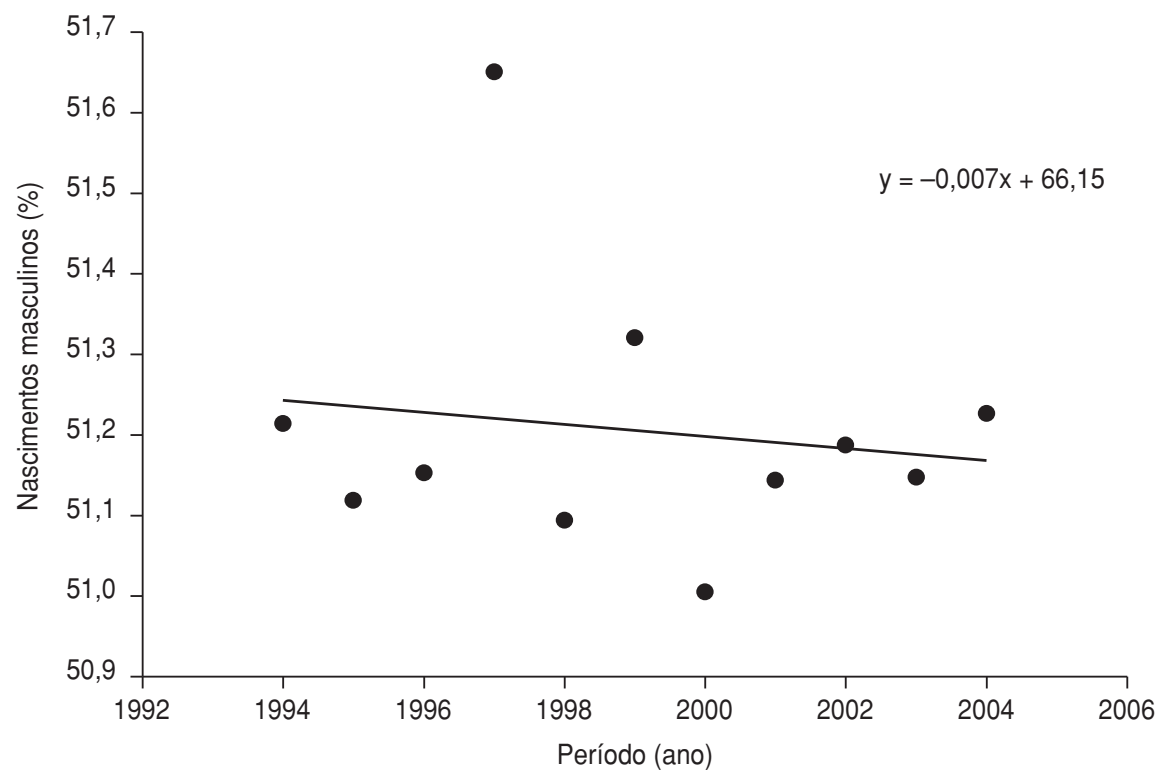

Dos 10 municípios mencionados, três apresentaram declínio significativo da proporção de nascimentos masculinos $(P<0,05)$ : Icaraíma, Palmeira e Barra do Jacaré (tabela 2). Em seguida, foi feito um diagrama de dispersão entre o consumo de agrotóxicos (1985) e a tendência da proporção masculina de nascimentos (1994 a 2004) para o grupo de 10 municípios. Embora tenha sido observada a existência de correlação negativa para as variáveis, esta não foi estatisticamente significativa (figura 1B).

A tabela 2 contém dados relativos a produção agrícola, porcentagem de trabalhadores na atividade agropecuária, número de estabelecimentos agropecuários, área colhida em hectare, consumo de agrotóxicos per capita e proporção de nascimentos masculinos nos anos de 1994 e 2004 nesses municípios, para efeitos comparativos. De acordo

TABELA 2. Perfil da atividade agrícola em $1996^{a}$ e do consumo de agrotóxicos em 1985 e proporção de nascimentos masculinos de 1994 a 2004 em municípios selecionados, Estado do Paraná, Brasil

\begin{tabular}{|c|c|c|c|c|c|c|c|}
\hline Município & $\begin{array}{c}\text { Pessoal } \\
\text { ocupado na } \\
\text { agropecuária, } \\
1996(\%)^{b}\end{array}$ & $\begin{array}{l}\text { No. } \\
\text { estabelecimentos } \\
\text { agropecuários, } \\
1996\end{array}$ & $\begin{array}{l}\text { Hectares } \\
\text { colhidos, } \\
1996\end{array}$ & $\begin{array}{l}\text { Principal tipo } \\
\text { de cultivo }\end{array}$ & $\begin{array}{l}\text { Agrotóxicos } \\
\text { per capita, } \\
1985 \text { (moeda } \\
\text { da época) }\end{array}$ & $\begin{array}{l}\text { Nascimentos } \\
\text { masculinos, } \\
\text { 1994(\%) }\end{array}$ & $\begin{array}{l}\text { Nascimentos } \\
\text { masculinos, } \\
2004(\%)\end{array}$ \\
\hline Santo Antônio do Caiuá & 14,2 & 65 & 87,2 & $\begin{array}{l}\text { Mandioca, algodão (herbáceo), } \\
\text { milho, feijão, soja, café }\end{array}$ & 130,4 & 62,5 & 43,8 \\
\hline Nova Aliança do Ivaí & 32,2 & 69 & 61,1 & $\begin{array}{l}\text { Cana-de-açúcar, mandioca, } \\
\text { sofá, milho, laranja, café }\end{array}$ & 95,9 & 50,0 & 50,0 \\
\hline Jardim Olinda & 14,2 & 31 & 5,4 & $\begin{array}{l}\text { Milho, soja, mandioca, algodão } \\
\text { (herbáceo), feijão, girassol, café }\end{array}$ & 147,7 & 62,5 & 26,3 \\
\hline Icaraíma ${ }^{\mathrm{C}}$ & 32,9 & 983 & 1016,8 & $\begin{array}{l}\text { Cana-de-açúcar, arroz, feijão, } \\
\text { milho, algodão, mandioca, } \\
\text { soja e café }\end{array}$ & 99,2 & 50,7 & 45,3 \\
\hline Cafeara & 31,9 & 181 & 179,5 & $\begin{array}{l}\text { Soja, milho, feijão, cana-de-açúcar, } \\
\text { algodão, café, girassol, amendoim }\end{array}$ & 541,8 & 50,7 & 40,0 \\
\hline Palmeira ${ }^{\mathrm{C}}$ & 23,4 & 2152 & 623,0 & $\begin{array}{l}\text { Milho, trigo, soja, feijão, fumo, } \\
\text { cevada (grão), aveia, batata } \\
\text { inglesa, arroz, erva-mate, maçã } \\
\text { e uva }\end{array}$ & 359,6 & 50,0 & 48,0 \\
\hline Barra do Jacaréc & 35,1 & 400 & 46,8 & $\begin{array}{l}\text { Cana-de-açúcar, café, arroz, } \\
\text { algodão, soja, milho e trigo }\end{array}$ & 449,0 & 59,3 & 52,0 \\
\hline
\end{tabular}

Fonte: Instituto Brasileiro de Geografia e Estatística (IBGE).

a Os dados de 1996 foram utilizados como indicadores indiretos da exposição a agrotóxicos.

b Os trabalhadores da agropecuária incluem homens, mulheres e crianças.

${ }^{c}$ Municípios com tendência significativa ao declínio na proporção de nascimentos masculinos $(\alpha=0,05)$. 
com esses dados, a variedade de cultivos não difere consideravelmente entre as localidades. Com exceção de Rio Bom, Nova Aliança do Ivaí e Barra do Jacaré, todos os municípios selecionados apresentaram proporção de nascimentos masculinos inferior a $50 \%$ em 2004, valores considerados não usuais, uma vez que a proporção de nascimentos masculinos deve ser superior a 51\% entre os nascidos vivos.

O mapa de Kernel estimado mostra a distribuição espacial segundo gradiente de consumo de agrotóxicos no Estado do Paraná como um todo. A partir dele, verificou-se que os $10 \mathrm{mu}-$ nicípios selecionados encontram-se localizados nas "regiões quentes" do mapa, dentro das faixas de maior consumo per capita de agrotóxicos, ao norte do Estado do Paraná (figura 3).

\section{DISCUSSÃO}

Apesar das evidências, os motivos pelos quais a proporção de nascimen- tos masculinos parece estar declinando em alguns países ainda não estão plenamente esclarecidos. Parece que ainda há muito que se aprofundar sobre o tema, que é relevante, porém relativamente novo e pouco explorado. Embora não haja conclusões definitivas sobre seus reais mecanismos de ocorrência, existem fortes evidências de que a redução de nascimentos de homens seja influenciada por exposições químicas ambientais e ocupacionais a desreguladores endócrinos provenientes de diferentes fontes de contaminação (20). Diversos estudos exemplificaram este efeito em populações expostas a dioxina $(4,11,12)$, pesticidas $(10,12,20)$ e bifenilas policloradas (21). A explicação é que a ação disruptora desses compostos sobre o sistema endócrino estaria alterando as concentrações hormonais das populações expostas, o que estaria provocando declínio no nascimento de homens em determinadas regiões. No entanto, alguns autores sugerem que não se pode atribuir a responsabilidade pelo declínio na proporção de nasci- mentos masculinos exclusivamente à poluição ambiental por desreguladores endócrinos, uma vez que não se sabe como se comportaria tal proporção na ausência desses poluentes (22).

Embora existam evidências crescentes de que a exposição ocupacional e ambiental a desreguladores endócrinos possa afetar a razão de sexos ao nascimento, os resultados obtidos até a presente data são de difícil interpretação, em virtude dos achados conflitantes e do grande número de variáveis envolvidas na determinação do sexo ao nascimento (14). Em contrapartida, estudos como o realizado por Figa-Talamanca, no qual se constatou uma associação significativa entre a exposição à dioxina, decorrente do acidente de Seveso em 1976, e o declínio na proporção de nascimentos de homens na coorte de estudo não devem ser desprezados (4).

Com base nessas evidências, buscamos informações sobre o comportamento da distribuição temporal da proporção de nascimentos masculinos

\section{FIGURA 3. Mapa de Kernel das regiões que tiveram maior intensidade de consumo de agrotóxicos em 1985 (hot spots), Es- tado do Paraná, Brasil}

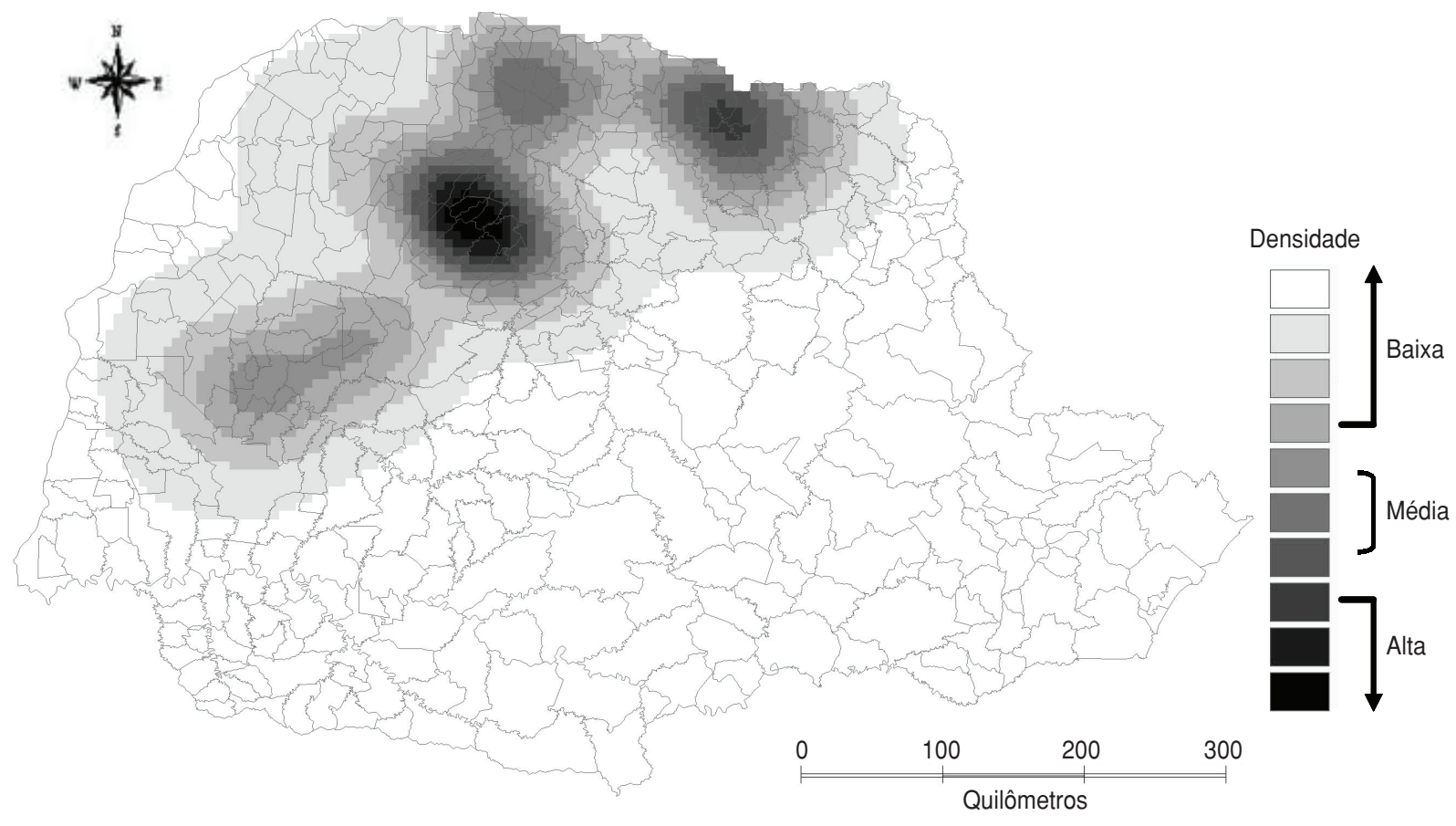


no Estado do Paraná, grande produtor rural e consumidor de agrotóxicos e produtos ligados à atividade agrícola (20). Os dados disponíveis sobre o consumo de agrotóxicos no ano de 1985 no Estado do Paraná nos permitiram realizar uma análise exploratória preliminar, na qual se buscou investigar a possível existência de correlação entre o referido consumo (exposição) e a tendência ao declínio de nascimentos masculinos na região. Embora não tenhamos observado uma correlação estatisticamente significativa entre as variáveis consumo de agrotóxicos e tendência da proporção de nascimentos masculinos, é conveniente que alguns pontos sejam considerados.

A prática de compra de agrotóxicos por um determinado município não significa necessariamente que o produto será para uso exclusivo no território do município comprador, fato que poderia introduzir tendenciosidade na interpretação dos resultados deste estudo. Outro ponto importante refere-se ao fato de as informações referentes ao consumo de agrotóxicos não estarem disponíveis segundo sua classificação por tipos específicos (inseticidas, herbicidas, fungicidas e outros), o que impede uma avaliação mais detalhada. A multiplicidade de propriedades e efeitos associados a essas diferentes classes de agrotóxicos resulta em dinâmicas diferenciadas nas matrizes ambientais e em diferentes graus de dano aos sistemas reprodutivos de animas e humanos (14).

Algumas características particulares dos municípios mereceriam ainda ser levadas em consideração, a exemplo da extensão territorial e da proximidade das áreas de cultivo dos centros urbanos, onde possivelmente encontra-se grande parte da população exposta, fator importante na caracterização da intensidade da exposição. Quanto a esse aspecto, é importante mencionar que se utilizou um grande número de municípios agrupados em quartis, cujo critério de formação baseou-se exclusivamente no consumo de agrotóxicos. Dessa forma, o presente estudo considerou o consumo de agrotóxicos como único indicador da exposição ambiental a desreguladores endócrinos, quando na verdade sabe-se que tais contaminantes podem ter diversas origens (20). Ao se agrupar em quartis um grande número de municípios, cada qual com geografia e economia próprias, é possível que tenha ocorrido uma diluição da correlação, fato que motivou a seleção e a análise individual de um grupo restrito de 10 municípios.

O gráfico de tendência linear dessas 10 localidades revelou um declínio visivelmente acentuado ao longo de apenas 11 anos (1994 a 2004). Nesses municípios, a proporção de nascimentos de crianças do sexo masculino passou de usual (acima de 51,0\%) a valores típicos de localidades que sofreram intensa exposição a desreguladores endócrinos (abaixo de 50\%) $(4,11,12)$.

A hipótese de que o uso abusivo de agrotóxicos tenha contribuído para o declínio de nascimentos de homens torna-se consistente quando analisamos o grau de proximidade entre os municípios, como mostra o mapa de Kernel - mais precisamente, uma localização dentro da área de influência calculada pelo estimador. Além disso, os diagramas de dispersão dos municípios pertencentes ao quarto quartil (figura 1A) (maiores consumidores de agrotóxicos) apresentaram tendência de declínio na proporção de nascimentos masculinos à medida que aumentaram os valores de consumo de agrotóxicos (embora sem significância estatística). Este é um forte indício de que o alto consumo de agrotóxicos nesse grupo de municípios possa estar influenciando a proporção de nascimentos de homens nessas localidades. É importante ressaltar ainda que os três municípios que apresentaram redução significativa de nascimentos de homens ao longo do período de estudo foram também os que apresentaram a maior área colhida em hectares (Icaraíma e Palmeira), a terceira maior porcentagem de trabalhadores agropecuários (Barra do Jacaré) e o maior número de estabelecimentos agropecuários (Palmeira e Icaraíma).
A limitação inerente aos estudos ecológicos quanto à dificuldade de estabelecer relações de causa e efeito constitui outro fato que deve ser considerado. Aliado a esse fato, acredita-se que a utilização da razão de sexos ao nascimento (expressa aqui na forma de proporção de nascimentos masculinos) como indicador sentinela da saúde reprodutiva e ambiental seja mais confiável quando aplicada a populações pequenas, especialmente quando a exposição a poluentes químicos é rigorosamente medida no indivíduo, o que não ocorre em estudos ecológicos.

\section{Considerações finais}

Os resultados analisados oferecem fortes evidências da provável participação do elevado consumo de agrotóxicos no declínio da proporção de nascimentos de crianças do sexo masculino no Estado do Paraná, sobretudo nos 10 municípios de pequeno porte selecionados.

Ao analisar o padrão da tendência de declínio nestes 10 municípios, verificou-se que o mesmo parece ser mais acentuado quando comparado ao padrão observado para o Estado do Paraná, sugerindo que a extensão territorial tende a diluir a velocidade com que tal declínio vem ocorrendo. Deve-se ressaltar ainda que as proporções de nascimentos masculinos nos municípios selecionados mostraram-se no limite inferior (boderline) em relação àquelas considerados usuais na literatura $(9,13)$. Quanto a isso, devem ser realizados novos estudos que englobem períodos mais extensos (considerando que o desfecho aqui estudado é produto de exposições crônicas), bem como estudos que avaliem a distribuição temporal de nascimentos masculinos em décadas anteriores à história de consumo excessivo de agrotóxicos nessas localidades. Isso permitiria uma avaliação mais precisa e segura desse indicador no Estado do Paraná, capaz de dimensionar a real magnitude do impacto do consumo de agrotóxicos na proporção de nascimentos masculinos. 


\section{REFERÊNCIAS}

1. Chahnazarian A. Determinants of the sex ratio at birth: review of recent literature. Soc Biol. 1988; 35:(3-4);214-35.

2. Schnorr TM, Lawson CC, Whelan EA. Spontaneous abortion, sex ratio and paternal occupational exposure to 2,3,7,8-tetrachlorodibenzop-dioxin. Environ Health Perspect. 2001; 109(11):1127-32.

3. Spira A, Multigner L. The effect of industrial and agricultural pollution on human spermatogenesis. Hum Reprod 1998;13(8):2041-2.

4. Figa-Talamanca I, Tarquini M, Lauria L. È possibile utilizzare il sex ratio alla nascita come indicatore di presenza di Endocrine Disruptors in contaminazioni ambientali? G Ital Med Lav Ergon. 2003;25 Suppl(3):52-3.

5. Brasília, Organização Pan-Americana de Saúde/Organização Mundial da saúde (OPAS/OMS). Indicadores básicos para a saúde no Brasil: conceitos e aplicações. Capítulo 3: fichas de qualificação dos indicadores, Brasília: OPAS. 2002. Disponível em: www. opas.org.br/sistema/arquivos/matriz.pdf. Acessado em maio de 2008.

6. Jongbloet PH, Zielhuis GA, Groenewoud MM, Pasker-de-Jong PCM. The secular trends in male:female ratio at birth in postwar industrialized countries. Environ Health Perspect. 2001;109(7):749-52.

7. Dickson HO, Parker L. Why is the sex ratio falling in England and Wales? J Epidemiol Community Health. 1996;(50):227-8.
8. Moller $\mathrm{H}$. Change in male: female ratio among newborn infants in Denmark. Lancet. 1996;348(9030):828-9.

9. Allan BB, Brant R, Seidel JE, Jarrel JF. Declining sex ratio in Canada. CMAJ. 1997;156(1):37-41.

10. Koifman S, Paumggartten FJ. Impact of environmental endocrine-active substances on public health. Cad Saude Publica. 2002;18(2): 354-5.

11. Mocarelli P, Gerthoux PM, Ferrari E, Patterson DG Jr, Kieszak SM, Brambilla P, et al. Paternal concentrations of dioxin and sex ratio of offspring. Lancet. 2000;355(9218):1858-63.

12. Ryan JJ, Amirova Z, Carrier G. Sex ratio of children on Russian pesticide producers exposed to dioxin. Environ Health Perspect. 2002;10(11):A699-701.

13. Jacobsen R, Moller $H$, Mouritsen A. Natural variation in the human sex ratio. Hum Reprod. 1999;14(12):3120-5.

14. Grisolia CK. Agrotóxicos: mutações, câncer e reprodução. Brasília: Universidade de Brasília; 2005.

15. Lemaire G, Terouanne B, Mauvais P, Michel $S$, Rahmani R. Effect of organochlorine pesticides on human androgen receptor activation in vitro. Toxicol Appl Pharmacol. 2004;196(2): 235-46.

16. Friedmann AS. Atrazine inhibition of testosterone production in rat males following peripubertal exposure. Reprod Toxicol. 2002; 16(3):275-9.
17. Oliva A, Spira A, Multigner L. Contribution of environmental factors to the risk of male infertility. Hum Reprod. 2001;16(8):1768-76.

18. Koifman S, Koifman RJ, Meyer A. Human reproductive system disturbances and pesticide exposures in Brazil. Cad Saude Publica. 2002; 18(2):435-45.

19. Rezende LPF, Parré JL. A hierarquização dos municípios paranaenses segundo as suas atividades agrícolas. Informe Gepec. 2003;7(2): 99-119. Disponível em: http://e-revista. unioeste.br/index.php/gepec/article/view/ 315. Acessado em maio de 2008.

20. Meyer A, Sarcinelli P, Moreira J. Are some Brazilian population groups subject to endocrine disrupters? Cad Saude Publica. 1999; 15(4):845-50.

21. Del Rio Gomez I, Marshall T, Tsai P, Shao YS Guo YL. Number of boys born to men exposed to polychlorinated byphenyls. Lancet. 2002;360(9327):143-4.

22. James WH. Was the widespread decline in sex ratios at birth caused by reproductive hazards? Hum Reprod. 1998;13(4):1083-4.

Manuscrito recebido em 5 de agosto de 2007. Aceito em versão revisada em 15 de fevereiro de 2008

ABSTRACT Objective. To determine the trend in male birth rates from 1994-2004 in the state of Paraná, Brazil, and whether a correlation exists between this trend and the state's agricultural toxic sales in 1985.

Agricultural toxic use and temporal distribution of male birth rate in the state of Paraná, Brazil

Method. This ecological study employed data from the Brazilian Institute for Geography and Statistics (agricultural toxic sales) and the Unified Health System's database "DATASUS" (sex ratio at birth). Three hundred and eight (308) municipalities were analyzed, divided into quartiles by pesticide usage. Each of the four resulting quartiles included 77 municipalities, with the fourth quartile representing the highest consumption. The male birth rate was obtained for each year for each municipality. Then the median rate was calculated for the entire period (1994-2004) for each municipality. A bivariate analysis was carried out. Pearson coefficients were calculated for each quartile. An exploratory and comparative analysis of quartiles was performed, and the mean per capita consumption was calculated for each quartile. Ten municipalities with intense agricultural activity were then selected and analyzed individually in terms of their agricultural profile and male birth rate trend during the study period.

Results. A discrete decline was observed in the rate of live male births for the entire state. The magnitude of this decline was more evident when the group of 10 municipalities was analyzed: in this group, the rates observed at the end of the study period were much lower than those usually described in the literature (male birth rate $<50 \%$ ). Pearson's correlation was negative for all quartiles, although without statistical significance.

Conclusions. The statistically significant trend towards a decline in male births in some of the Paraná municipalities suggests that this phenomenon may be a result of a high level of environmental exposure to pesticides. Future studies should confirm and measure the impact of environmental pesticide contamination on reproductive health, expressed in this study as sex ratio at birth.

\footnotetext{
Key words Pesticides, environmental pollution, reproductive medicine, Brazil.
} 